\title{
Periprostatic schwannoma
}

\section{mimicking metastatic lymphadenopathy in a case of multifocal prostate adenocarcinoma}

\author{
Jia Wei Tan ${ }^{1}$, Jyothirmayi Velaga ${ }^{1}$, John Shyi Ping Yuen ${ }^{2}$, Xin Min Cheng ${ }^{3}$, Yan Mee Law ${ }^{1}$ \\ 1. Department of Diagnostic Radiology, Singapore General Hospital, Singapore \\ 2. Department of Urology, Singapore General Hospital, Singapore \\ 3. Department of Anatomical Pathology, Singapore General Hospital, Singapore
}

* Correspondence: Jia Wei Tan, Department of Diagnostic Radiology, Singapore General Hospital, Outram Rd, 169608, Singapore (入tanjiawei@hotmail.com)

Radiology Case. 2021 Mar; 15(3):9-18 :: $\quad$ DOI: 10.3941/jrcr.v15i3.4210

\begin{abstract}
Schwannomas of the prostate are a rare entity and usually diagnosed incidentally following surgical management of presumed benign prostate hyperplasia or prostate adenocarcinoma. We present a case of sporadic periprostatic schwannoma diagnosed in conjunction with multifocal prostate adenocarcinoma on pre-operative multiparametric magnetic resonance imaging.
\end{abstract}

\section{CASE REPORT}

\section{CASE REPORT}

A 67-year-old male presented to the urology clinic for lower urinary tract symptoms consisting of increased frequency of urination and nocturia. Additionally, he had a raised serum prostate specific antigen (PSA) level of 12.2 UG/L (normal range: $0-4.0$ ) and a raised PSA density of 0.24 UG/L/ML. Digital rectal examination was unremarkable and transabdominal bedside ultrasound revealed a prostate volume of $40 \mathrm{ml}$ with intravesical protrusion. He also had a history of erectile dysfunction with an International Index of Erectile Function (IIEF) score of 12 out of 30.

The finding of a raised serum PSA level prompted further investigation with multiparametric magnetic resonance imaging (mpMRI) of the prostate. Anatomical imaging comprised of T1-weighted (T1w) axial sequences of the pelvis as well as high-resolution T2-weighted (T2w) sequences of the prostate in the axial, coronal and sagittal planes, with functional imaging consisting of diffusion weighted (DWI) sequences (high b-value up to $1800 \mathrm{~s} / \mathrm{mm} 2$ ) and dynamic contrast enhanced (DCE) sequences (temporal resolution 5s).

mpMRI revealed a prostate gland volume of $51.8 \mathrm{ml}$. There were two dominant T2w hypointense lesions with marked restricted diffusion and early intense enhancement (PIRADS 5) in both lobes of the prostate gland (Fig. 1 and 3). The PIRADS 5 lesion involving the right lobe anterior peripheral and transition zones at the mid gland measured 2.1 $\mathrm{x} 4.7 \times 1.6 \mathrm{~cm}$ with no evidence of extraprostatic extension. The PIRADS 5 lesion in the left lobe posterior peripheral zone at the apex and mid gland measured $1.6 \times 1.4 \times 1.7 \mathrm{~cm}$ with broad contact $(1.5 \mathrm{~cm})$ to the prostatic capsule, but no tumour extension into the periprostatic fat. DCE imaging revealed early enhancement of the left neurovascular bundle, suspicious for neurovascular bundle infiltration. There was an additional $1.8 \times 1.5 \times 1.3 \mathrm{~cm}$ well-circumscribed, mild and heterogeneous $\mathrm{T} 2 \mathrm{w}$ hypointense lesion in the left periprostatic region adjacent to the neurovascular bundle and abutting the 
prostatic capsule (Fig. 2 and 3). This lesion demonstrated restricted diffusion and mild heterogeneous enhancement.

Subsequent transperineal MRI-ultrasound fusion targeted biopsy revealed multiple cores of Gleason $4+4$ adenocarcinoma from MRI-identified lesions in both lobes of the prostate gland. The patient was offered robotic assisted laparoscopic radical prostatectomy for definitive treatment with en-bloc resection of the periprostatic lesion. Intraoperative findings included an approximately $2 \mathrm{~cm}$ rounded, well circumscribed extra-prostatic lesion just caudal to the left vascular pedicle at the prostate-vesical junction (Fig. 4) which appeared to be embedded within the neurovascular bundle. The lesion was excised en-bloc with the neurovascular tissue in a non-nerve sparing fashion given the radiological finding of probable left neurovascular bundle infiltration by tumour.

Whole mount histopathological examination of the resected specimen revealed pT2 Gleason 4+4 adenocarcinoma (grade group 4) in both lobes of the prostate gland with a small tertiary component of Gleason pattern 5; the latter was 1 $\mathrm{mm}$ in size, admixed with pattern 4 structures, and consisted of cords and occasionally singly dispersed tumour cells. There was perineural invasion but no extraprostatic extension and surgical margins were negative. The periprostatic lesion showed discrete, well-circumscribed spindle cell proliferation with hypercellular and some hypocellular areas. Cells in hypercellular areas contained wavy nuclei with nuclear palisading, while the hypocellular areas were more myxoid in appearance. There was mild to moderate cytological atypia, but the cells showed fine and evenly distributed nuclear chromatin pattern throughout with a very low mitotic rate (up to 1 per 10 high power fields). The atypia was thus attributed to ancient change. No necrosis was identified. The lesion was positive for SOX10 and S100 on immunohistochemistry, with some patchy weak SMA staining focally. Immunohistochemistry CD117, caldesmon, desmin, AE1/3, EMA, and DOG-1 were negative. Histopathological findings were consistent with a schwannoma (Fig. 5).

The patient was well post-operatively and prescribed a 3month course of tadalafil $5 \mathrm{mg}$ as part of penile rehabilitation. However, he saw no improvement to his erectile function and declined further treatment for it. He was continent preoperatively and regained full continent control by two months post-operatively. There was no biochemical evidence of tumour recurrence at one-year follow-up, i.e. serum PSA level was not elevated.

\section{DISCUSSION}

\section{Etiology \& Demographics:}

Schwannomas are benign mesenchymal tumours originating from the Schwann cells of the peripheral nerve sheaths. They can occur at all ages but are most frequent in persons 20 to 50 years old [1]. They classically display a typical biphasic pattern on histology with areas of hypercellularity (Antoni type A) and hypocellularity, the latter within a myxoid stroma (Antoni type B) [1]. These lesions are usually solitary and sporadic but may also occur in association with syndromes such as NF1, NF2, Carney complex and schwannomatosis [1]. The head, neck, and flexor surfaces of the upper and lower extremities are the sites most commonly affected [1]. Schwannomas of the prostate are a rare entity in patients with neurofibromatosis and are even rarer in patients without, such as in this case, with less than 10 documented incidents of prostatic or periprostatic schwannomas in PubMed-indexed literature to date [2-6].

\section{Clinical \& Imaging Findings:}

In our case, the proximity of the schwannoma to the PIRADS 5 lesion in the left lobe posterior peripheral zone at the apex and mid gland raised suspicion for tumour involvement. However, its circumscribed and distinct outline was atypical for a malignant process and its signal and enhancement pattern also differed from the primary prostate tumour. For example, the schwannoma demonstrated mild and heterogeneous $\mathrm{T} 2 \mathrm{w}$ hypointensity with mild heterogenous enhancement. This was in contrast to the primary prostate tumour, which showed marked and homogeneous T2w hypointensity with early intense enhancement. Restricted diffusion reflects hypercellularity and can be seen in schwannomas; it may not necessarily represent malignancy. T2w hypointensity is unusual in schwannomas but may reflect the presence of haemosiderin or calcification. The usage of Ga-PSMA PET-CT would not have been useful in this scenario, as schwannomas may also show PSMA uptake [7].

This case is unique as we have an MRI examination providing superior morphological, anatomical and functional information about the incidentally detected periprostatic schwannoma, for which there is a dearth of literature concerning the MRI features. Most described cases of prostatic schwannoma are often within the prostate gland, and imaging features would likely overlap with the heterogeneous MRI appearance of benign prostate hyperplastic nodules in the transition zone. The unique location in the periprostatic soft tissue implies that the schwannoma was most likely arising from the posterolateral neurovascular bundle.

\section{Treatment \& Prognosis:}

Radiological interpretation of periprostatic lesions is important for clinical decision-making as a diagnosis of locally advanced or metastatic disease may deprive the patient of a chance at curative surgical treatment, as opposed to a diagnosis without. Similarly, a diagnosis of neurovascular bundle infiltration by the tumour would necessitate non-nerve sparing surgery with the latter affecting the patient's erectile function and quality of life.

Schwannomas are generally benign and the prognosis is favourable if there is no evidence of malignant degeneration [1]. Surgical resection is curative but may not be necessary in the absence of symptoms, with active surveillance being a viable alternative.

Theoretically, there would have been an option for nervesparing surgery and active surveillance of the periprostatic schwannoma if our patient had not had evidence of neurovascular bundle infiltration on MRI. 


\section{Differential Diagnosis:}

There is bias towards diagnosing periprostatic lesions found on MRI as lymphadenopathy or extraprostatic extension of disease in the setting of prostate carcinoma. This is doubly so when the lesion in question arises close to or within the neurovascular bundle, a structure often involved by tumour in cases of locally advanced disease.

Periprostatic lymphadenopathy can be due to metastatic disease or reactive hyperplasia. Determination of metastatic lymph nodes via CT or MRI is largely by size criteria, with thresholds of $1.0 \mathrm{~cm}$ in short axis for oval nodes and $0.8 \mathrm{~cm}$ for round nodes recognised as indicators of probable metastatic disease [8]. However, usage of size criteria alone can limit sensitivity as more than half of metastatic lymph nodes may be below the size threshold while non-metastatic nodes may be enlarged due to reactive hyperplasia [9]. Malignant nodes have similar tissue properties to the primary tumour and tend to demonstrate restricted diffusion; ADC values are usually lower in malignant lymph nodes as opposed to benign nodes $[10,11]$. Other features such as a rounded shape, irregular border, hypointense $\mathrm{T} 2 \mathrm{w}$ signal and early intense enhancement also support the diagnosis of metastatic disease $[12,13]$

Extraprostatic extension (EPE) of prostate cancer refers to the presence of tumour beyond the confines of the prostate gland and is important to identify on pre-operative imaging given the surgical implications of the finding and the adverse prognosis associated with it. Morphological predictors of EPE on MRI include broad-based capsular contact length of $15 \mathrm{~mm}$ or greater by the tumour, capsular irregularity or bulge, frank breach of capsule and asymmetric neurovascular bundle thickening [14]. It is also contiguous with and inseparable from the primary lesion with similar imaging characteristics such as markedly hypointense $\mathrm{T} 2 \mathrm{w}$ signal and intense early enhancement. The affected neurovascular bundle is usually thickened and shows early linear enhancement with restricted diffusion.

Ectopic prostatic tissue is an uncommon manifestation that has been observed in other structures of the genitourinary tract such as the urethra, urinary bladder, testis, epididymis and seminal vesicles $[15,16]$. Its origin is unclear, with several theories having been proposed to explain this phenomenon. These include migration of normal tissue, persistence of embryonic remnants, and metaplastic change caused by chronic inflammation $[17,18]$. Being histologically indistinguishable from normal prostatic tissue, it can subsequently undergo non-malignant adenomatous overgrowth, i.e. benign prostate hyperplasia (prostate adenoma). On MRI, ectopic prostate adenomas are wellcircumscribed when interpreted in the axial, coronal and sagittal planes, clearly distinguishing it from EPE of prostate cancer. There is variable $\mathrm{T} 2 \mathrm{w}$ signal intensity with early intense enhancement and mild restricted diffusion.

Ultimately, the definitive diagnosis of an atypical periprostatic lesion such as a schwannoma is histopathological. However, a careful approach and interpretation of MRI findings, with special attention paid to the border contour, morphology, MR signal and enhancement pattern of the lesion will allow for the sensible formulation of alternate differential radiological diagnoses. In our case, the two main features that guided our differential diagnoses were the well-circumscribed appearance of the periprostatic lesion as well as the presence of restricted diffusion within it.

\section{TEACHING POINT}

Periprostatic schwannomas are well circumscribed, encapsulated and have a distinct margin separate from the prostate. They show mild to heterogeneous $\mathrm{T} 2 \mathrm{~W}$ hypointense signal and lack of early intense enhancement on dynamic contrast administration. In contrast, perineural invasion from prostate adenocarcinoma is contiguous with and inseparable from the primary tumour. The affected neurovascular bundle is usually thickened and shows early linear enhancement with restricted diffusion.

\section{REFERENCES}

1. Weiss SW, Goldblum JR, Folpe AL. Benign Tumors of Peripheral Nerves. In: Enzinger and Weiss's soft tissue tumors 7th ed. Elsevier Health Sciences; 2019 Nov 11; 913-918. ISBN: 0323610978, 9780323610971

2. Francica G, Bellini S, Miragliuolo A. Schwannoma of the prostate: ultrasonographic features. Eur Radiol. 2003 Aug;13(8):2046-8. PMID: 12942306.

3. Rane A, Juhasz A, McEwan A, Mene A. A urological diagnostic conundrum: schwannoma masquerading as an enlarged prostate. Br J Urol. 1995 May;75(5):683-4. PMID: 7542135

4. Üçer O, Fatih Zeren M, Fatih Kuyumcuo?lu M, Lekili M. Schwannoma of the prostate: a rare case report and literature review. Gen Med Open Access. 2016;3(6). Available at: https://doi.org/10.4172/2327-5146.1000219. Accessed July 22,2020

5. Jiang R, Chen JH, Chen M, Li QM. Male genital schwannoma, review of 5 cases. Asian J Androl. 2003 Sep;5(3):251-4. PMID: 12937811.

6. Dietrick B, Friedes C, White MJ, Allaf ME, Meyer AR. Incidental periprostatic schwannoma discovered during evaluation for prostatic adenocarcinoma. Urol Case Rep. 2020 Feb 29;31:101150. PMID: 32154115

7. Dias AH, Bouchelouche K. Prostate-Specific Membrane Antigen PET/CT Incidental Finding of a Schwannoma. Clin Nucl Med. 2018 Apr;43(4):267-268. PMID: 29401152.

8. Bellin MF, Roy C, Kinkel K, et al. Lymph node metastases: safety and effectiveness of MR imaging with ultrasmall superparamagnetic iron oxide particles--initial clinical experience. Radiology. 1998;207:799-808. PMID: 9609907 
9. Davis GL. Sensitivity of frozen section examination of pelvic lymph nodes for metastatic prostate carcinoma. Cancer. 1995;76:661-668. PMID: 8625162

10. Eiber M, Beer AJ, Holzapfel K, et al. Preliminary results for characterization of pelvic lymph nodes in patients with prostate cancer by diffusion-weighted MR-imaging. Invest Radiol. 2010;45:15-23. PMID: 19996762

11. Akduman EI, Momtahen AJ, Balci NC, et al. Comparison between malignant and benign abdominal lymph nodes on diffusion-weighted imaging. Acad Radiol. 2008;15:641-646. PMID: 19996762

12. Thoeny HC, Froehlich JM, Triantafyllou M, et al. Metastases in normal-sized pelvic lymph nodes: detection with diffusion-weighted MR imaging. Radiology. 2014;273:125135. PMID: 24893049

13. Magnetta, M.J., Catania, R., Girometti, R. et al. Prostate MRI: staging and decision-making. Abdom Radiol 45, $2143-$ 2153 (2020). PMID: 32047994

14. Park KJ, Kim MH, Kim JK. Extraprostatic Tumor Extension: Comparison of Preoperative Multiparametric MRI Criteria and Histopathologic Correlation after Radical Prostatectomy. Radiology. 2020 Jul;296(1):87-95. PMID: 32368959.

15. Halat S, Eble JN, Grignon DJ, at al. Ectopic prostatic tissue: histogenesis and histopathological characteristics. Histopathology. 2011 Apr;58(5):750-8. PMID: 21438904.

16. Tan, FQ., Xu, X., Shen, BH. et al. An unusual case of retrovesical ectopic prostate tissue accompanied by primary prostate cancer. World J Surg Onc 10, 186 (2012). PMID: 22966979

17. Wallace C, Creager AJ, Cappellari JO, et al. Ectopic prostatic tissue in the uterine cervix. Am J Surg Pathol. 2001, 25 (9): 1215-1216. PMID: 11688585

18. Roy C, Guth S, Gasser B, et al. Benign hyperplasia in ectopic prostatic tissue: a rare cause of pelvic mass. Eur Radiol. 1997, 7 (1): 35-37. 10.1007/s003300050104. PMID: 11688585 


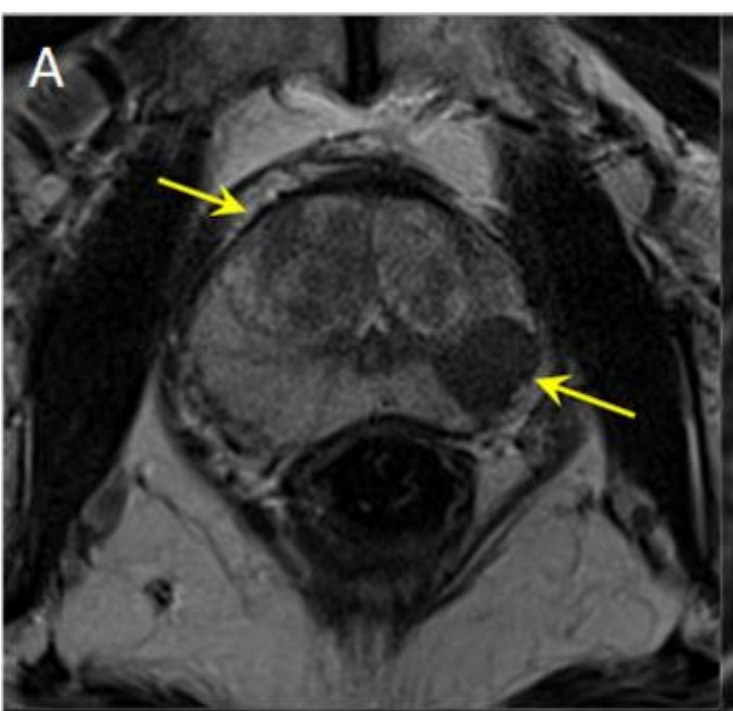

B
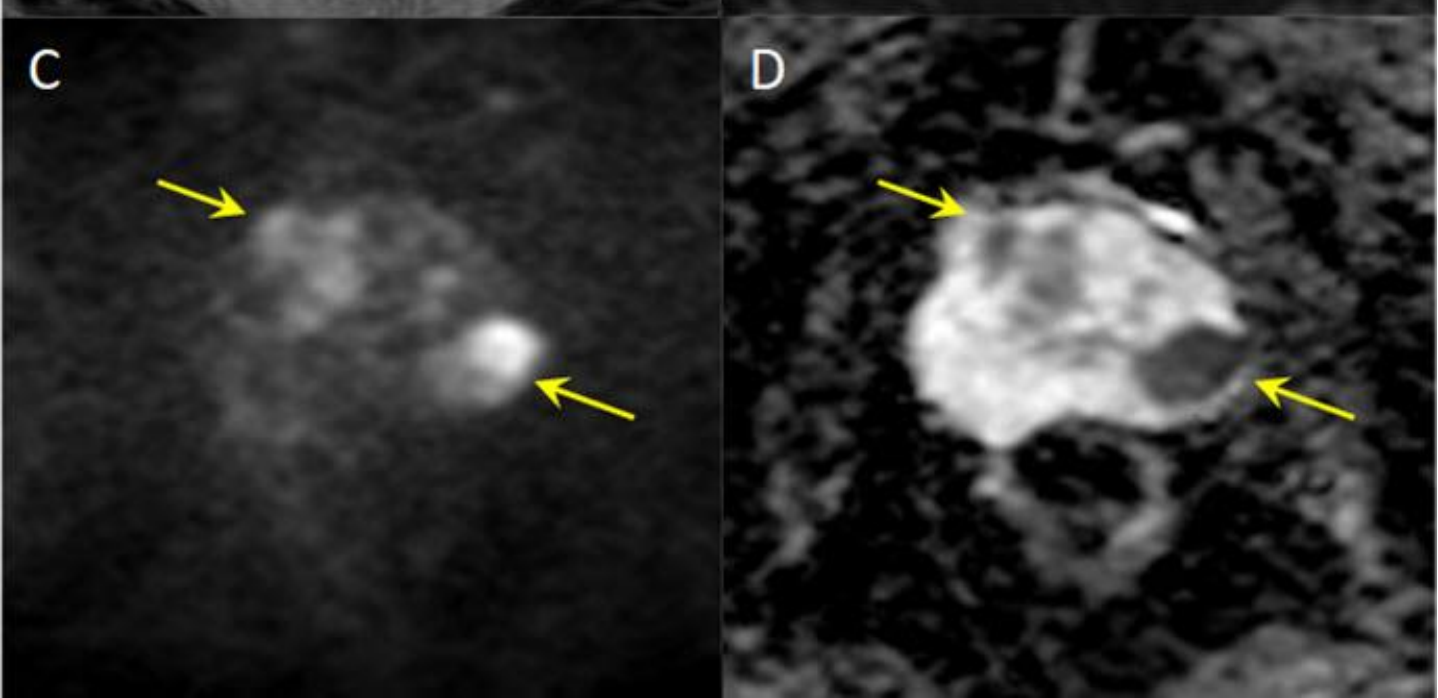

Figure 1: 67-year-old man with a periprostatic schwannoma diagnosed in conjunction with multifocal prostate carcinoma.

FINDINGS: Axial T2w image (A) demonstrates T2w hypointense PIRADS 5 lesions in the right anterior peripheral zone and transition zone as well as in the left posterior peripheral zone at the mid gland (arrows), measuring $2.1 \times 4.7 \times 1.6 \mathrm{~cm}$ and $1.6 \mathrm{x}$ $1.4 \times 1.7 \mathrm{~cm}$, respectively. Both lesions show avid enhancement on the axial T1w fat-saturated DCE sequence (arrows in B) as well as marked restricted diffusion on the axial DWI (C) and ADC (D) sequences (arrows). Axial T1w fat-saturated DCE sequence (B) also demonstrates early linear enhancement of the left neurovascular bundle (dashed circle), indicative of left neurovascular bundle involvement.

TECHNIQUE: Siemens Skyra MRI scanner. Magnetic strength 3 Tesla.

A: Axial T2-weighted, TR $3230 \mathrm{~ms}$, TE $101 \mathrm{~ms}$, slice thickness $3 \mathrm{~mm}$

B: Axial DCE, TR $3.86 \mathrm{~ms}$, TE $1.27 \mathrm{~ms}$, slice thickness $3 \mathrm{~mm}$, Dotarem $16.6 \mathrm{ml}, 51.4 \mathrm{~s}$ post-injection

C: Axial DWI, TR $3200 \mathrm{~ms}$, TE $84 \mathrm{~ms}$, slice thickness $3 \mathrm{~mm}$, b-value 1800

D: Axial ADC, TR $3200 \mathrm{~ms}$, TE $84 \mathrm{~ms}$, slice thickness $3 \mathrm{~mm}$ 


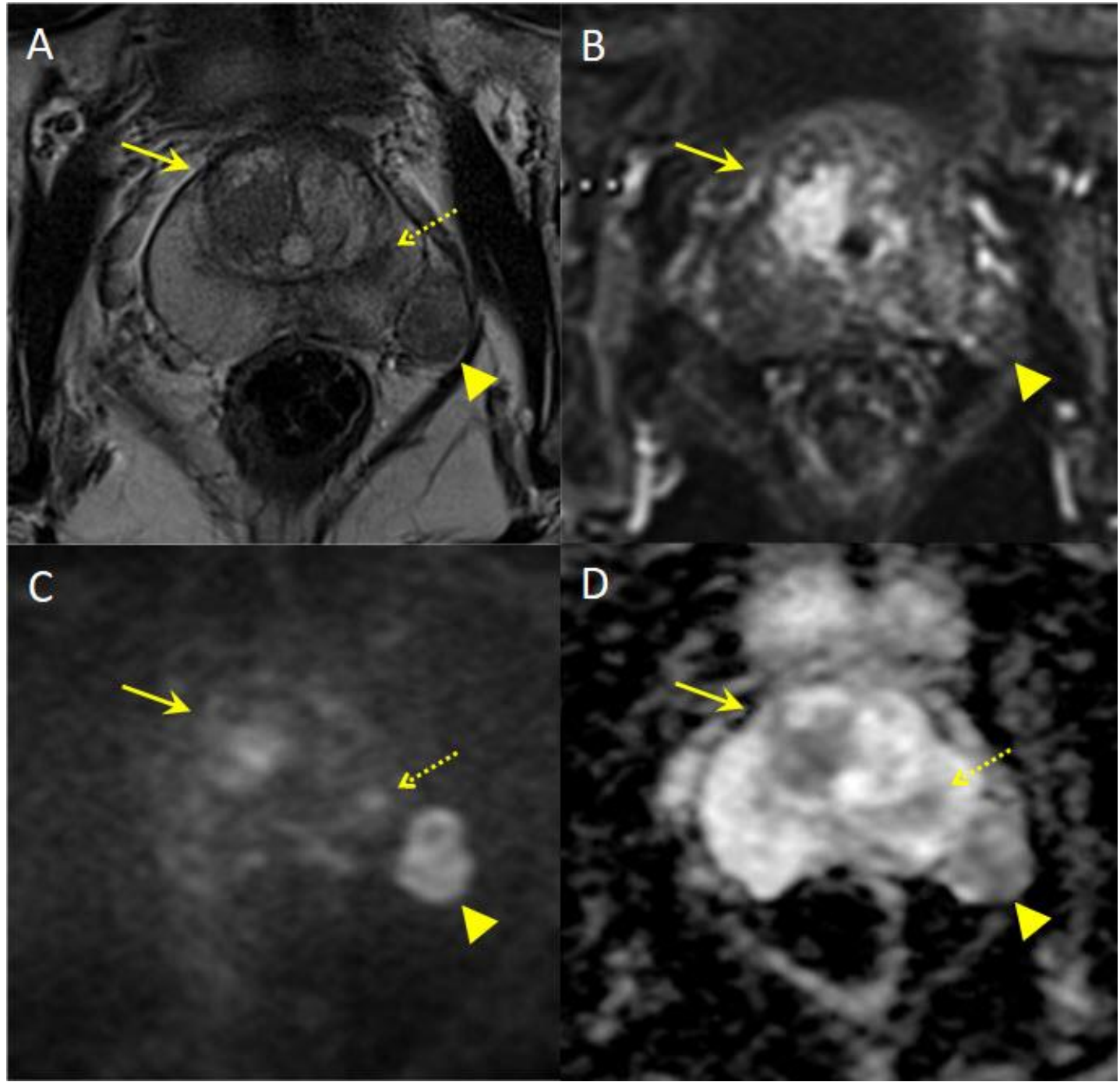

Figure 2: 67-year-old man with a periprostatic schwannoma diagnosed in conjunction with multifocal prostate carcinoma.

FINDINGS: Axial T2w sequence (A) demonstrates a 1.8 x 1.5 x $1.3 \mathrm{~cm}$ circumscribed, heterogeneous T2w hypointense periprostatic lesion (arrowhead), the right transition zone PIRADS 5 lesion (arrow) and the partially imaged left peripheral zone PIRADS 5 lesion (dashed arrow). The axial T1w fat-saturated DCE sequence (B) demonstrates differential enhancement between the periprostatic lesion (arrowhead) and the right transition zone PIRADS 5 lesion (arrow) with the latter showing more avid enhancement compared with the former. Axial DWI (C) and ADC (D) sequences demonstrate restricted diffusion in both the periprostatic lesion (arrowhead) and the PIRADS 5 lesions (arrow, dashed arrow).

TECHNIQUE: Siemens Skyra MRI scanner. Magnetic strength 3 Tesla.

A: Axial T2-weighted, TR $3230 \mathrm{~ms}$, TE $101 \mathrm{~ms}$, slice thickness $3 \mathrm{~mm}$

B: Axial DCE, TR $3.86 \mathrm{~ms}$, TE $1.27 \mathrm{~ms}$, slice thickness $3 \mathrm{~mm}$, Dotarem $16.6 \mathrm{ml}, 74.2 \mathrm{~s}$ post-injection

C: Axial DWI, TR $3200 \mathrm{~ms}$, TE $84 \mathrm{~ms}$, slice thickness $3 \mathrm{~mm}$, b-value 1800

D: Axial ADC, TR $3200 \mathrm{~ms}$, TE $84 \mathrm{~ms}$, slice thickness $3 \mathrm{~mm}$ 


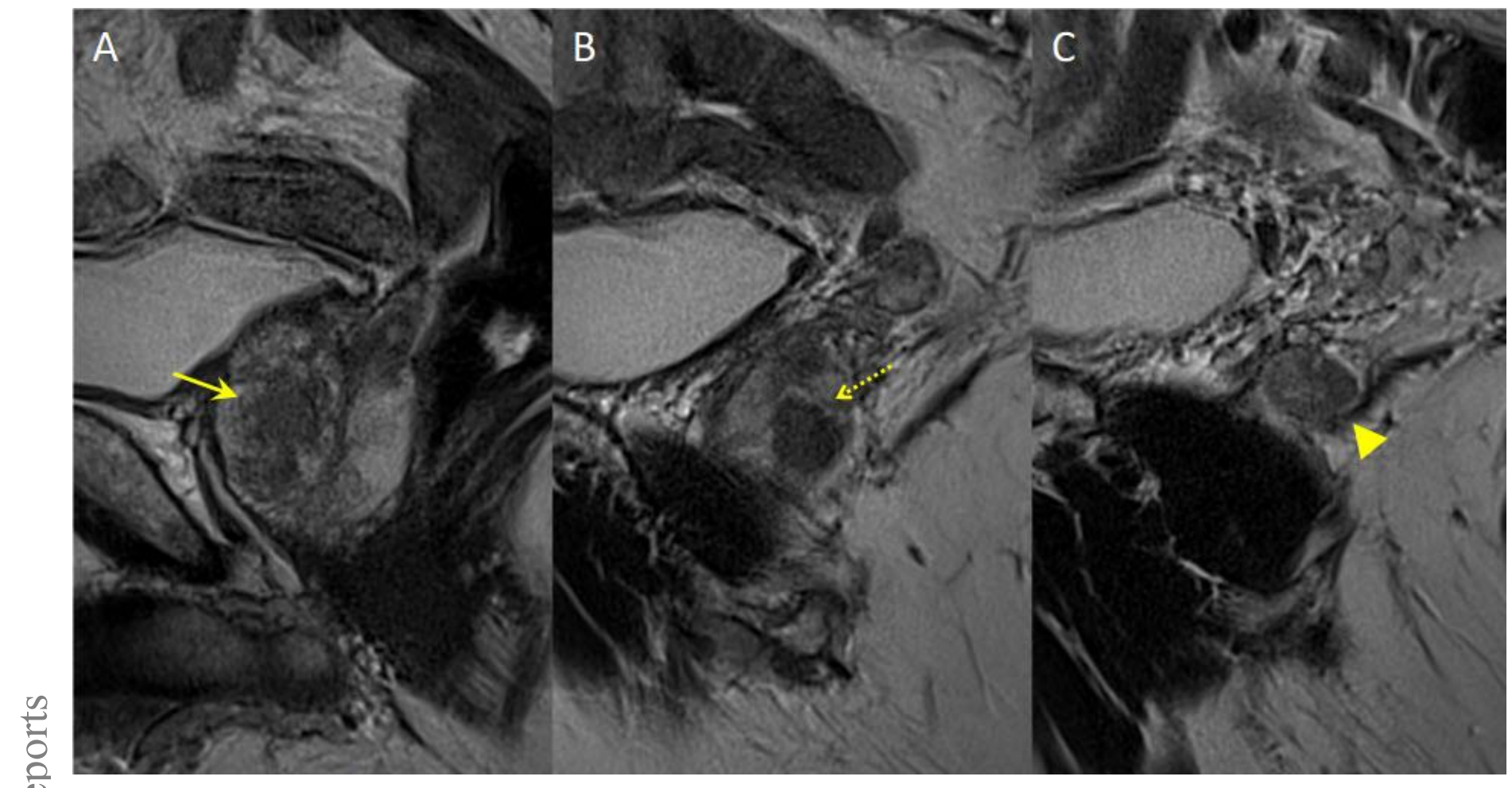

Figure 3: 67-year-old man with a periprostatic schwannoma diagnosed in conjunction with multifocal prostate carcinoma.

FINDINGS: Sagittal T2w sequences demonstrate T2w hypointense PIRADS 5 lesions in the right anterior peripheral zone and transition zone (arrow in A) as well as in the left posterior peripheral zone at the mid gland (dashed arrow in B). There is also a circumscribed, heterogeneous T2w hypointense lesion in the left periprostatic region (arrowhead in C).

TECHNIQUE: Siemens Skyra MRI scanner. Magnetic strength 3 Tesla.

A, B and C: Sagittal T2-weighted, TR $3980 \mathrm{~ms}$, TE $101 \mathrm{~ms}$, slice thickness $3 \mathrm{~mm}$

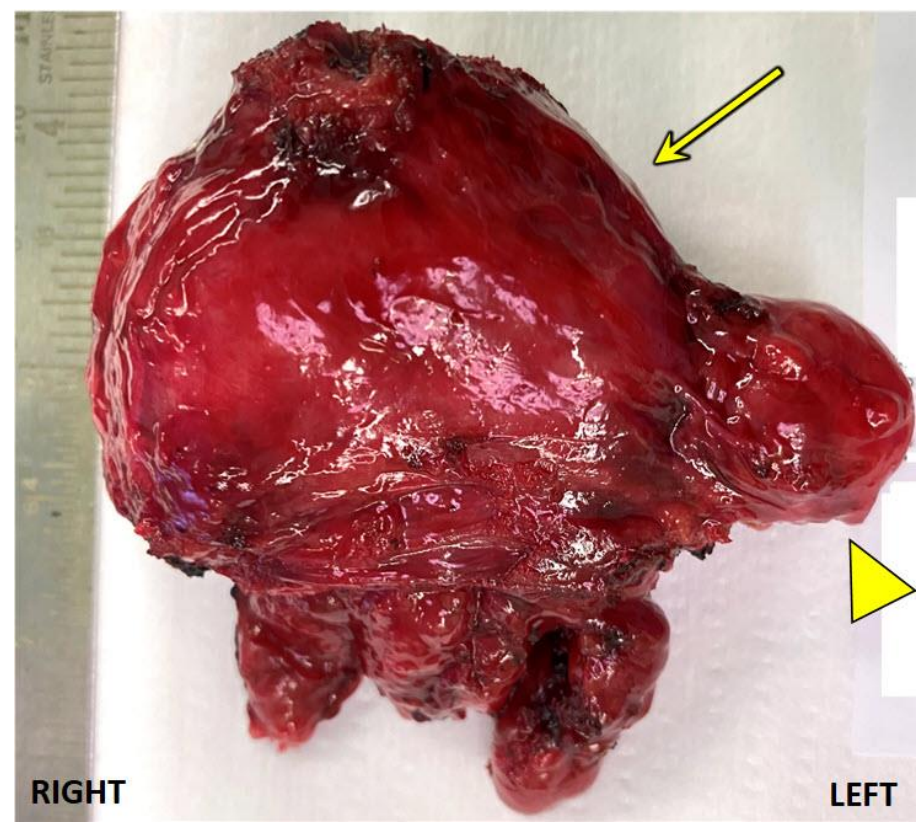

Figure 4 (left): 67-year-old man with a periprostatic schwannoma diagnosed in conjunction with multifocal prostate carcinoma, status post-radical prostatectomy with enbloc resection of the periprostatic lesion.

FINDINGS: High-resolution macroscopic image of the operative specimen shows the prostate (arrow) and an approximately $2.0 \mathrm{~cm}$ rounded, well circumscribed extraprostatic lesion (arrowhead) just caudal to the left vascular pedicle at the prostate-vesical junction; the latter appeared to be embedded within the neurovascular bundle. 


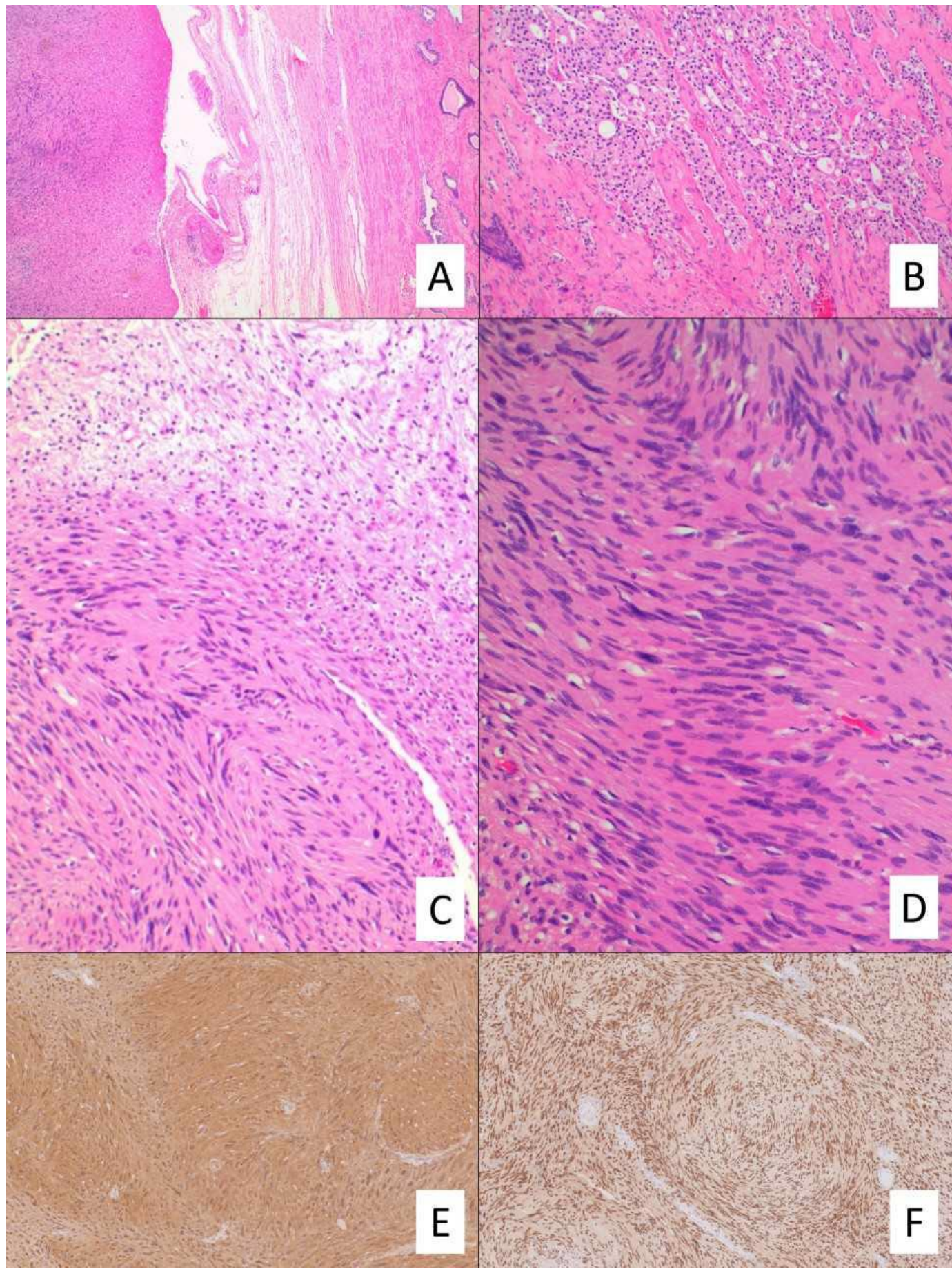

Figure 5: 67-year-old man with a periprostatic schwannoma diagnosed in conjunction with multifocal prostate carcinoma. Digital photographs of the pathology slides from the operative specimen.

FINDINGS: Image (A) shows a discrete, circumscribed spindle cell lesion on the left and the adjacent prostate on the right, separated by loose connective tissue. Image (B) demonstrates Gleason 4+4 acinar adenocarcinoma in the prostate. Image (C) of the spindle cell lesion shows hypocellular (top) and hypercellular (bottom) areas with some atypia attributable to ancient change. Image (D) of the spindle cell lesion shows a hypercellular area with prominent nuclear palisading. Images (E) and (F) show that the spindle cell lesion cells are positive for S100 and SOX10 stains respectively, further supporting the diagnosis of a schwannoma.

\section{TECHNIQUE:}

A: Hematoxylin and eosin stain (H\&E stain; 40X)

B, C and D: Hematoxylin and eosin stain (H\&E stain; 100X)

E: Immunohistochemical staining for $\mathrm{S} 100$

F: Immunohistochemical staining for SOX10 


\begin{tabular}{|l|l|}
\hline Etiology & Encapsulated nerve sheath tumour of Schwann cell origin. \\
\hline Incidence & Rare, given the dearth of cases published in PubMed-indexed literature. \\
\hline Gender ratio & Males only \\
\hline Age predilection & $2^{\text {nd }}$ to $5^{\text {th }}$ decade \\
\hline Risk factors & Syndromes such as NF1, NF2, Carney complex and schwannomatosis \\
\hline Treatment & Surgical excision if symptomatic; role for active surveillance if asymptomatic \\
\hline Prognosis & Favourable if no evidence of malignant degeneration \\
\hline Imaging findings & $\begin{array}{l}\text { Gross: focal, well-circumscribed lesion } \\
\text { T1: isointense to prostate parenchyma }\end{array}$ \\
& T2: heterogenous signal with possible cystic areas \\
& DCE: mild enhancement \\
& DWI/ADC: may show restricted diffusion depending on degree of hypercellularity. \\
\hline
\end{tabular}

Table 1: Summary table of periprostatic schwannoma.

\begin{tabular}{|c|c|c|c|}
\hline & Etiology & MRI Findings & Management \\
\hline $\begin{array}{l}\text { Extraprostatic } \\
\text { extension of } \\
\text { disease }\end{array}$ & Local spread of tumour & $\begin{array}{l}\text { Ill-defined, contiguous, and inseparable from the } \\
\text { primary tumour. Involved area demonstrates } \\
\text { similar imaging characteristics to the primary } \\
\text { tumour, namely marked T2w hypointensity with } \\
\text { early intense enhancement. The affected } \\
\text { neurovascular bundle is usually thickened and } \\
\text { shows early linear enhancement with restricted } \\
\text { diffusion. }\end{array}$ & $\begin{array}{l}\text { Malignant } \\
\text { En-bloc excision if patient } \\
\text { is for surgical management; } \\
\text { multi-modal therapy is an } \\
\text { option otherwise. }\end{array}$ \\
\hline Schwannoma & $\begin{array}{l}\text { Primary nerve sheath } \\
\text { tumour }\end{array}$ & $\begin{array}{l}\text { Well circumscribed, encapsulated and has a } \\
\text { distinct margin separate from the prostate. } \\
\text { Shows mild to heterogeneous T } 2 \mathrm{~W} \text { hypointense } \\
\text { signal and lack of early intense enhancement on } \\
\text { dynamic contrast administration }\end{array}$ & $\begin{array}{l}\text { There is a role for active } \\
\text { surveillance if } \\
\text { asymptomatic. Surgery is } \\
\text { curative. }\end{array}$ \\
\hline $\begin{array}{l}\text { Enlarged lymph } \\
\text { node }\end{array}$ & $\begin{array}{l}\text { Malignant infiltration or } \\
\text { reactive enlargement }\end{array}$ & $\begin{array}{l}\text { Malignant } \\
\text { Usually shows restricted diffusion and an ill- } \\
\text { defined contour. Signal and enhancement pattern } \\
\text { reflect that of the primary tumour. In the case of } \\
\text { prostate carcinoma, it would be markedly } \mathrm{T} 2 \mathrm{w} \\
\text { hypointense with early intense enhancement. } \\
\text { Reactive } \\
\text { Usually circumscribed and oval-shaped with } \\
\text { homogenous signal intensity and no restricted } \\
\text { diffusion. }\end{array}$ & $\begin{array}{l}\text { Malignant } \\
\text { En-bloc excision if patient } \\
\text { is for surgical management; } \\
\text { multi-modal therapy is an } \\
\text { option otherwise. } \\
\frac{\text { Reactive }}{\text { Nil treatment required. }}\end{array}$ \\
\hline $\begin{array}{l}\text { Ectopic prostate } \\
\text { adenoma }\end{array}$ & $\begin{array}{l}\text { Non-malignant } \\
\text { adenomatous growth of } \\
\text { ectopic prostatic tissue }\end{array}$ & $\begin{array}{l}\text { Well-defined and well circumscribed when } \\
\text { interpreted in axial, coronal, and sagittal planes. } \\
\text { Variable T2w signal intensity. May show early } \\
\text { intense enhancement and mild restricted } \\
\text { diffusion. }\end{array}$ & $\begin{array}{l}\text { Managed medically as per } \\
\text { benign prostate hyperplasia. }\end{array}$ \\
\hline
\end{tabular}

Table 2: Differential diagnosis table for a periprostatic lesion with restricted diffusion on a background of prostate carcinoma. 


\section{ABBREVIATIONS}

$\mathrm{ADC}=$ Apparent diffusion coefficient

DCE $=$ Dynamic contrast enhanced

DWI $=$ Diffusion-weighted imaging

$\mathrm{EPE}=$ Extraprostatic extension

Ga-PSMA = Gallium-prostate specific membrane antigen mpMRI = Multiparametric magnetic resonance imaging

PET-CT = Positron Emission Tomography - Computed

Tomography

PIRADS = Prostate imaging reporting and data system

PSA $=$ Prostate specific antigen

$\mathrm{T} 2 \mathrm{w}=\mathrm{T} 2$-weighted

\section{KEYWORDS}

Periprostatic; Schwannoma; Prostate; Adenocarcinoma; Lymphadenopathy

\section{Online access}

This publication is online available at:

www.radiologycases.com/index.php/radiologycases/article/view/4210

\section{Peer discussion}

Discuss this manuscript in our protected discussion forum at: www.radiolopolis.com/forums/JRCR

\section{Interactivity}

This publication is available as an interactive article with scroll, window/level, magnify and more features.

Available online at www.RadiologyCases.com

Published by EduRad

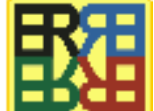

www.EduRad.org 